\title{
Night sky brightness simulation over Montsec protected area
}

\author{
Hector Linares ${ }^{\mathrm{a}, \mathrm{b}}$, Eduard Masana ${ }^{\mathrm{a}}$, Salvador J. Ribas ${ }^{\mathrm{b}, \mathrm{a}}$, Martin Aubéc ${ }^{\mathrm{c}}$, Alexandre \\ Simoneau ${ }^{\mathrm{d}}$, Salvador Baráe \\ ${ }^{a}$ Institut de Ciències del Cosmos (ICC-UB-IEEC), Barcelona, Spain \\ ${ }^{b}$ Parc Astronòmic Montsec, Ager, Spain \\ ${ }^{c}$ Département de physique, Cégep de Sherbrooke, Sherbrooke, Canada \\ ${ }^{d}$ Université de Sherbrooke, Sherbrooke, Canada \\ ${ }^{e}$ Universidade de Santiago de Compostela, Santiago de Compostela, Galicia
}

\begin{abstract}
Night sky brightness over Montsec Observatory (north-east of Spain) has been computed and checked against measurements using Illumina numerical model (Aubé, 2007). In a previous publication (Linares et al., 2018) the methodology was validated and light pollution received in the observatory coming from a unique city was computed. Here we present a simulation that includes all the sources that has a significant impact over the quality of the night sky in this area.

The decision of which sources should be included in the simulations was taken following the methodology explained by Bará and Lima (2018): using a point spread function (PSF) as a simple approach to estimate which sources are brightening the sky dome over the observer. An ad hoc PSF derived with Illumina was used with the purpose of avoiding to have to rely on already existing empirical PSF. The resulting PSF can be used in any location with similar atmospheric conditions. Differences in the spectrum of the lamps can be accounted easily by adjusting a spectrum scale factor.

Illumina simulates the artificial sky brightness received $(\mathrm{W} / \mathrm{sr} / \mathrm{m} 2)$ by an observer from any direction. Adding the natural sky brightness allows to compare the simulations to measurements taken with different instrumentation. In our case simulations were checked against ASTMON, SQC and SQM measurements. They show a good agreement both in absolute values and in geographical patterns for the three filters studied, B, V and R.

The methodology presented opens many possibilities, such as increasing the reliability of the maps that point out the light pollution main contributors for any location, and reducing the amount of time needed to perform an accurate simulation of the night sky brightness.
\end{abstract}

Preprint submitted to Journal of Quantitative Spectroscopy and Radiative TransferMarch 16, 2020 
Keywords: light pollution, modelling, psf, Illumina

\section{Introduction}

Light pollution can be understood as the misuse of artificial lighting. It includes excessive intensity, illuminating beyond the target area, in hours when there is no need and choosing poorly the color of the light emitted.

Concerns about the darkness of the night have been a common topic in astronomic facilities for decades. A clear atmosphere is by itself an obstacle for observing the cosmos, that's why it is necessary to rely on observations from satellites to widen the range of the electromagnetic spectrum that can be studied. Light pollution worsens considerably the problem, to the extent that many observatories on Earth have become completely useless for scientific purposes.

Nonetheless, light pollution should not be taken as a problem solely for astronomers. It affects the vast majority of flora and fauna that lived for millennia according to the light cycle of day and night and the variations of light exposure throughout the year. Humans are no exception, links between light pollution and some diseases have been showed recently (Garcia-Saenz et al., 2018). Moreover, it contributes indirectly to climate change through wasting energy that is mostly produced by fossil fuel combustion. Beyond that reasoning and from a romantic perspective, it would be amazing to be able to gaze at the cosmos from many more locations than today.

The goal of the present work is to find a light pollution prediction tool that can simulate measurements in a reasonable timescale and with validated precision. A reliable simulation tool might simplify, reduce the cost and speed up light pollution studies by overcoming frequent obstacles when measuring as inaccessible locations or bad weather conditions.

In this sense there are two light pollution numerical models that standout from previous ones for accounting heterogeneity of light sources spectrum and optical features: $\mathrm{MSNsR}_{\mathrm{Au}}$ (Kocifaj, 2007), and Illumina (Aubé et al., 2005, 2018). The first one requires less CPU time but it does not account for second scattering. It is specially useful when studying nearby sources with optically thin atmopheres. Illumina on the other hand accounts for first and second order scattering, that makes it very time consuming but suitable for cases that include faraway sources as is the case of this study. These two models in their early stage versions have been compared in a study in the Canary Islands (Aubé and Kocifaj, 2012).

The area chosen to test the methodology is Montsec mountain range in the northeast of Spain. It is an astronomical protected area labeled as Reference Point ac- 
cording to Law 6/2001 of Government of Catalonia due to its pristine conditions and also certified as Starlight Reserve. It hosts a scientific observatory, Parc Astronòmic Montsec (PAM), which is the location for the simulations. It is a perfect place to test the methodology due to the amount of measurements taken from there and the vast information available about the light inventories from the surrounding towns. This area has been chosen recently to study the effects of different atmospheric conditions over artificial light propagation (Jechow et al., 2018, 2017).

The first simulations of the night sky brightness maps over PAM were showed in Linares et al. (2018). They included the city of Lleida as the unique light source. The results were encouraging and the process was validated. Here simulations are improved by including any light pollution source in a radius of $50 \mathrm{~km}$ around the PAM and any major light pollution contributor outside this zone.

Including more sources means increasing the accuracy of the results as well as increasing the time needed to preform the simulations. The CPU time, which is proportional with the amount of light pollution sources that are included, normally extends to hundreds of hours. Including every light pollution source in the calculus is not an option for most of the cases when working on light pollution. One strategy to reduce the computing time is to know beforehand which sources ought to be included in the calculus without decimating the accuracy of the results. In this sense Bará and Lima (2018) showed a very convenient way to achieve this knowledge. They use satellite image to know the luminous flux for each location and an empirical point spread function $^{1}$ (PSF) derived by Duriscoe et al. (2018) to know which towns and cities are the main contributors to light pollution over the location studied.

However, this PSF is not suitable for our case for various reasons: 1) is derived using the city of Las Vegas (USA) as a source, with an inventory of lights completely different from the one present in the towns around the PAM; 2) the atmospheric conditions from the Arizona desert, where the experiment was performed, does not apply for the Pyrenees area; 3) it only defines the filter $\mathrm{V}$ of the Johnson-Cousins photometric system (Johnson and Morgan, 1953, 1955; Cousins, 1976) that leave out necessary information that can be achieved studying also B and $\mathrm{R}$.

To solve these problems the idea of using Illumina to obtain a new PSF that fits the case came up. It allows to set atmospheric conditions, define the inventory of lights and study the range of the spectrum desired. The resultant PSF is defined in a way that can be used in any other location with similar atmospheric conditions and for different wavelength ranges within 350 and $830 \mathrm{~nm}$. In the case that the lamps

\footnotetext{
${ }^{1}$ The PSF refelcts how light propagates through the atmosphere. It depends on variables such as atmospheric conditions and spectrum of the light sources
} 
used in the zone to study are different than the ones present in Montsec the user only have to adjust the scale factor related with the spectrum of the light sources (see subsection 3.4).

This paper is structured as follows: in section 2 it is explained how to obtain a PSF using Illumina, in section 3 are shown the resulting maps with the contribution by municipalities, and in section 4 the advanced night sky brightness maps of Montsec protected area along with their comparison with measured values.

\section{Illumina $\mathbf{v} 1$}

Illumina v1 is a sophisticated light propagation numerical model able to simulate with precision the human induced sky radiance. It takes into account first and second order molecular sacattering and aerosol scattering and absortion both direct and after reflecting on the ground (see figure 1). The model doesn't account for molecular absorption but as long as the wavelength range studied is within the visible range, this absorption is negligible (Gueymard, 2001).

The user can choose which light sources to include in the calculations. It is done defining circular areas by a central point and a radius. Every point of light within one of these circular areas will be taken into account. The characteristics of the points of light in each circular area are defined homogeneously. That is why normally big cities are defined in an independent circular area, they can even be split in several areas if the characteristics of the lamps differ between regions inside the city. On the other hand smaller and similar towns can be grouped together. To characterize each area the following features must be defined: lamp spectra, light output angular distribution with respect to the vertical axis, lamp height respective to the ground, obstacle's size and distance, and the probability for a photon of hitting an obstacle. Normally these properties are the mean or typical values of the points of light within each area, it is of great help having access to the detailed inventory of the lighting systems of cities and towns. The intensity of the light is not uniform over each area, this irregularity is taken into account using satellite data from the VIIRS-DNB sensor.

The model also takes into account of the geographical characteristics of the environment (elevation and reflectivity of the ground) by using a digital elevation model and satellite spectral albedo information. It also uses multiple parameters to define the atmosphere: humidity, aerosol model, aerosol optical depth, Angstrom coefficient and sea level pressure.

To compute sky brightness in a given direction, Illumina divides the atmosphere in $3 \mathrm{D}$ cells and takes the sum over the cells along the line of sight ( $n$ in figure 1$)$ 


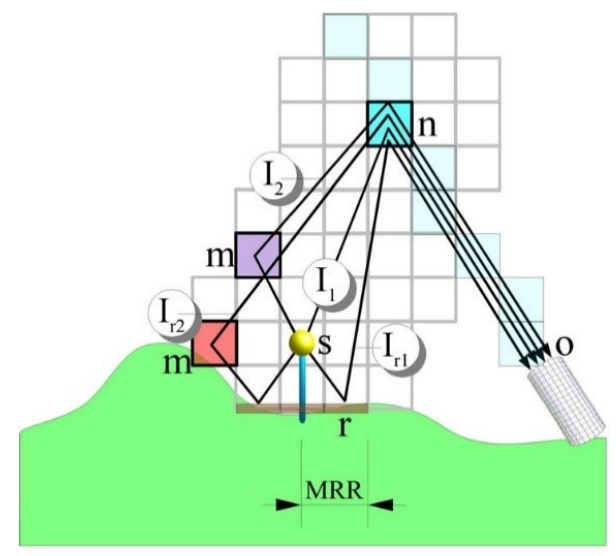

Figure 1: Scheme of the model used by ILLUMINA to compute the flux received in each direction. $\mathrm{S}$, source of light; O, observer's location; $\mathrm{n}$, any 3D cell in the line of sight of the observer; $\mathrm{m}$, cells that scatter light to $\mathrm{n} ; I_{1}$, first order scattering path; $I_{2}$, second order scattering path; $I_{r 1}$, reflection and first order scattering path; $I_{r 2}$, reflection and second order scattering path; r, reflection on the ground; MRR, maximum radius taken into account for the reflection. Image from Aubé et al. (2005).

to integrate the total light that arrives to the observer in that direction. The model assume that the total flux is mainly explained by the combination of the first-order scattering ( $I_{1}$ and $I_{r 1}$ in figure 1$)$ and the second-order scattering $\left(I_{2}\right.$ and $I_{r 2}$ in figure $1)$.

Aerosol and molecular light extinction arising during various light paths between the light source and the observer is also computed. It is assumed that the vertical profiles of the molecular and aerosol concentrations follow exponentially decreasing functions. It is also assumed that aerosol and molecular concentration profiles are uniform over the modeling domain. For further details see Aubé et al. (2005).

\section{Deriving a PSF using Illumina v1}

The PSF describes the behaviour of the light emitted by a point like source through the atmosphere and estimates the radiance received from it in any location at distance d. Bará and Lima (2018) showed how to use it to show which sources and in which percentage are polluting the sky over a specific location. This information is useful to decide which light sources have to be included in a simulation.

There exists different PSFs in the bibliography accounting for the propagation of light through the atmosphere, most of them empiric. Which means that they are defined by the conditions of the place and date where the measurements took place. 
The purpose here is to use a simplified scenario in Illumina v1 to replace the empiric measurements for deriving a PSF.

The simplified scenario consists in:

- Unique point like light source

- Flat topography

- Homogeneous light reflection on the ground

- Typical atmospheric conditions of the area

- Clear sky, absence of clouds

\subsection{Point light source}

The source of light is defined to have the minimum surface area to simulate a point light source. By the time this study was performed, the minimum size of a light source allowed by Illumina $\mathrm{v} 1$ was the corresponding of one pixel of the satellite images from the VIIRS-DNB sensor. In our case it is close to $1 \times 1 \mathrm{~km}$.

With the purpose to be able to work with a non-monochromatic PSF, applicable to any inventory of lights, $16 \mathrm{PSF}$ are computed covering the range from $350 \mathrm{~nm}$ to $830 \mathrm{~nm}$ in windows of $30 \mathrm{~nm}$. The point-like source is defined by a spectrum with 16 peaks every $30 \mathrm{~nm}$ that coincides with the division of the spectrum used for each PSF. This way the 16 PSF can be combined to fit any light source spectrum. This range, as stated, includes the $\mathrm{B}, \mathrm{V}$ and $\mathrm{R}$ filters.

Other parameters needed to compute the PSF (as a matter of fact any calculus with Illumina) are: 1)the angular distribution, the pattern that describes how the light escapes from the lamp, and 2)obstacle characterization, distance to blocking elements, their heights and the probability of the light to be blocked. Angular distribution and obstacle characterisation have to be fixed. Therefore, oppositely of what happens with the inventory that will be needed only in the last step of the process, the PSF is linked from the beginning to these features. In the case of study, the typical values for the towns and cities around the PAM has been used: upward light output radio (ULOR) 10\%, height of the lamps 6m, obstacle's height $6 \mathrm{~m}$, probability of the photons to get trough obstacle $50 \%$ and horizontal distances from lamps to obstacles $5 \mathrm{~m}$. 


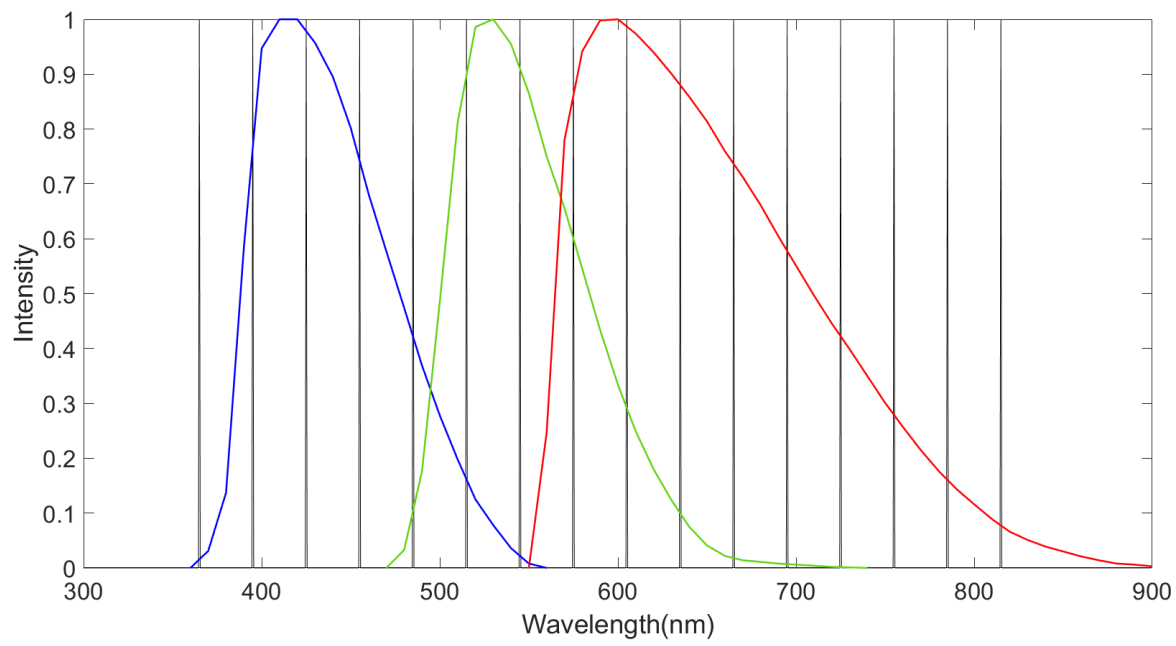

Figure 2: Spectrum used for point light source (black line). It is a discrete spectra with a peak in the center of the 16 wavelengths windows that cover the range of the $\mathrm{B}$ (blue line), $\mathrm{V}$ (green line) and $\mathrm{R}$ (red line) filters.

\subsection{Scenario characterisation}

As previously stated Illumina takes into account topography and ground reflection. When deriving a PSF it cannot be linked with these two features because it is going to be used in different directions and location, light will face different obstacles and grounds in each case. For that reason a flat terrain has been adopted with a constant reflection value approximated to the mean value found in rural areas.

As for the atmosphere characterization it has been assumed a rural environment with a relative humidity of $70 \%$, atmospheric standard pressure $(101,3 \mathrm{kPa}$ at sea level) and clear sky (no clouds). Typical aerosol optical depth (AOD) and angstrom coefficients (AC) are taken from the data from AERONET (AErosol RObotic NETwork, Holben et al. (1998)): AOD 0.09 and AC 1.0.

The distance range goes as far as $200 \mathrm{~km}$ from the light source. Beyond this distance is very unusual that light sources pollute significantly the sky. Huge cities could often be seen farther away but only in elevated observation points and only close to the horizon (even below it) in the direction pointing to the source.

Illumina provided the radiance received at each distance, for every direction in azimuth and elevation and for each subdivisions (colors) of the spectrum (16 windows of $30 \mathrm{~nm}$ ). The radiance for the whole dome has been integrated because the purpose is to study the contribution of the light sources in all directions. Other indicators 


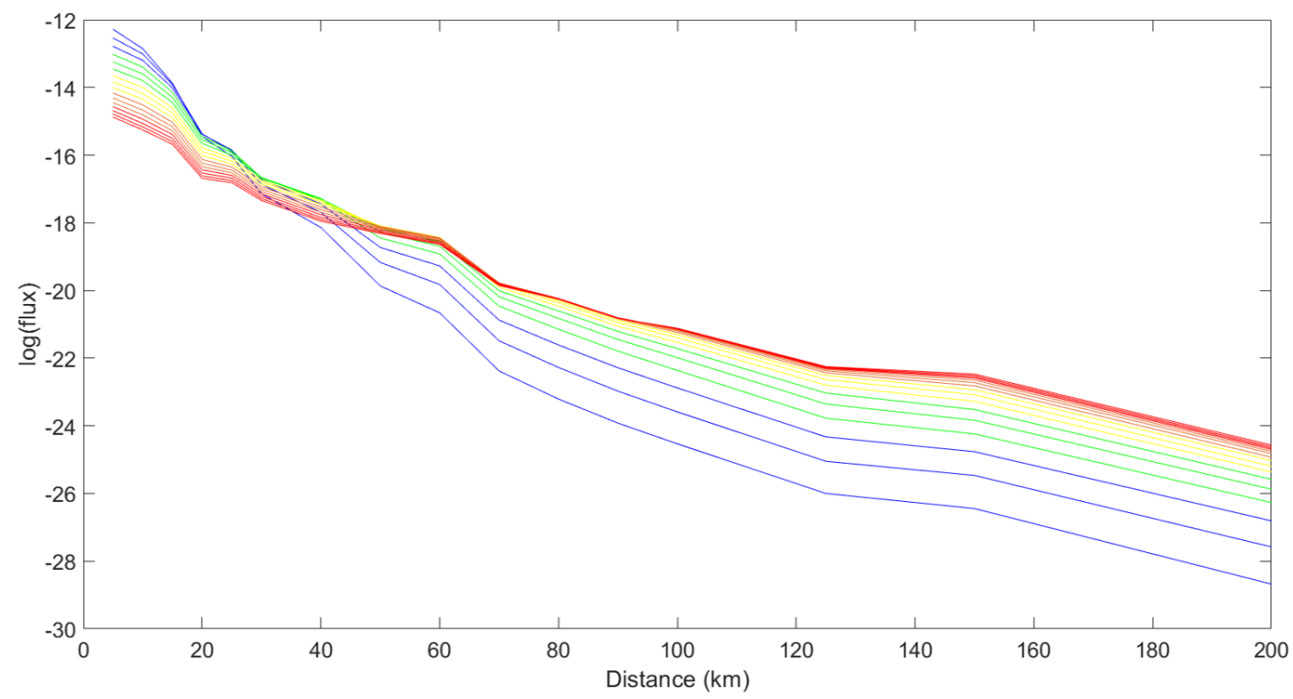

Figure 3: Radiance $\left(\mathrm{Wsr}^{-1} \mathrm{~m}^{-2}\right)$ received from the point-like source in each band with respect to the distance of the observer

could be used in other studies, such as the ring between 0 and 30 in elevation for biology studies or the dome over 30 for observation studies in astronomy, (see also Duriscoe (2016)).

In figure 3 is shown how the integrated radiance in the dome of the 16 different colors behave differently with distance. Bluer light is more polluting in short distances and have a steeper decay with distance than redder light, that is the least polluting in short distances but its flatter decay makes it the bigger problem in large distances.

\subsection{Fitting the data to a curve}

The final PSF is the result of fitting the data shown in figure 3 to a mathematical function. The purposes are having the same kind of expression for each one of the 16 spectral window as and being reliable for the entire distance range. After observing the data an exponential curve for each spectral window is the best match.

Being $\mathrm{d}$ the distance and $\mathrm{F}$ the function that describes radiance, the curves take the form:

$$
F(d)=10^{a d^{2}+b d+c}
$$




\begin{tabular}{|c|c|c|c|}
\hline Center wavelength & $\mathrm{a}$ & $\mathrm{b}$ & $\mathrm{c}$ \\
\hline 365 & -5.0958 & $-7.7321 \mathrm{e}-02$ & $2.0988 \mathrm{e}-04$ \\
395 & -5.2148 & $-6.8715 \mathrm{e}-02$ & $1.8016 \mathrm{e}-04$ \\
425 & -5.3438 & $-6.1841 \mathrm{e}-02$ & $1.5633 \mathrm{e}-04$ \\
455 & -5.4710 & $-5.6384 \mathrm{e}-02$ & $1.3751 \mathrm{e}-04$ \\
485 & -5.5909 & $-5.2069 \mathrm{e}-02$ & $1.2288 \mathrm{e}-04$ \\
515 & -5.7026 & $-4.8617 \mathrm{e}-02$ & $1.1144 \mathrm{e}-04$ \\
545 & -5.8079 & $-4.5850 \mathrm{e}-02$ & $1.0254 \mathrm{e}-04$ \\
575 & -5.8995 & $-4.3625 \mathrm{e}-02$ & $9.5512 \mathrm{e}-05$ \\
605 & -5.9828 & $-4.1882 \mathrm{e}-02$ & $9.0599 \mathrm{e}-05$ \\
635 & -6.0625 & $-4.0353 \mathrm{e}-02$ & $8.6015 \mathrm{e}-05$ \\
665 & -6.1336 & $-3.9139 \mathrm{e}-02$ & $8.2864 \mathrm{e}-05$ \\
695 & -6.1997 & $-3.8026 \mathrm{e}-02$ & $7.9649 \mathrm{e}-05$ \\
725 & -6.2583 & $-3.7128 \mathrm{e}-02$ & $7.7250 \mathrm{e}-05$ \\
755 & -6.3163 & $-3.6303 \mathrm{e}-02$ & $7.5009 \mathrm{e}-05$ \\
785 & -6.3660 & $-3.5582 \mathrm{e}-02$ & $7.3107 \mathrm{e}-05$ \\
815 & -6.4086 & $-3.4976 \mathrm{e}-02$ & $7.1683 \mathrm{e}-05$ \\
\hline
\end{tabular}

Table 1: Coefficients of the curves 

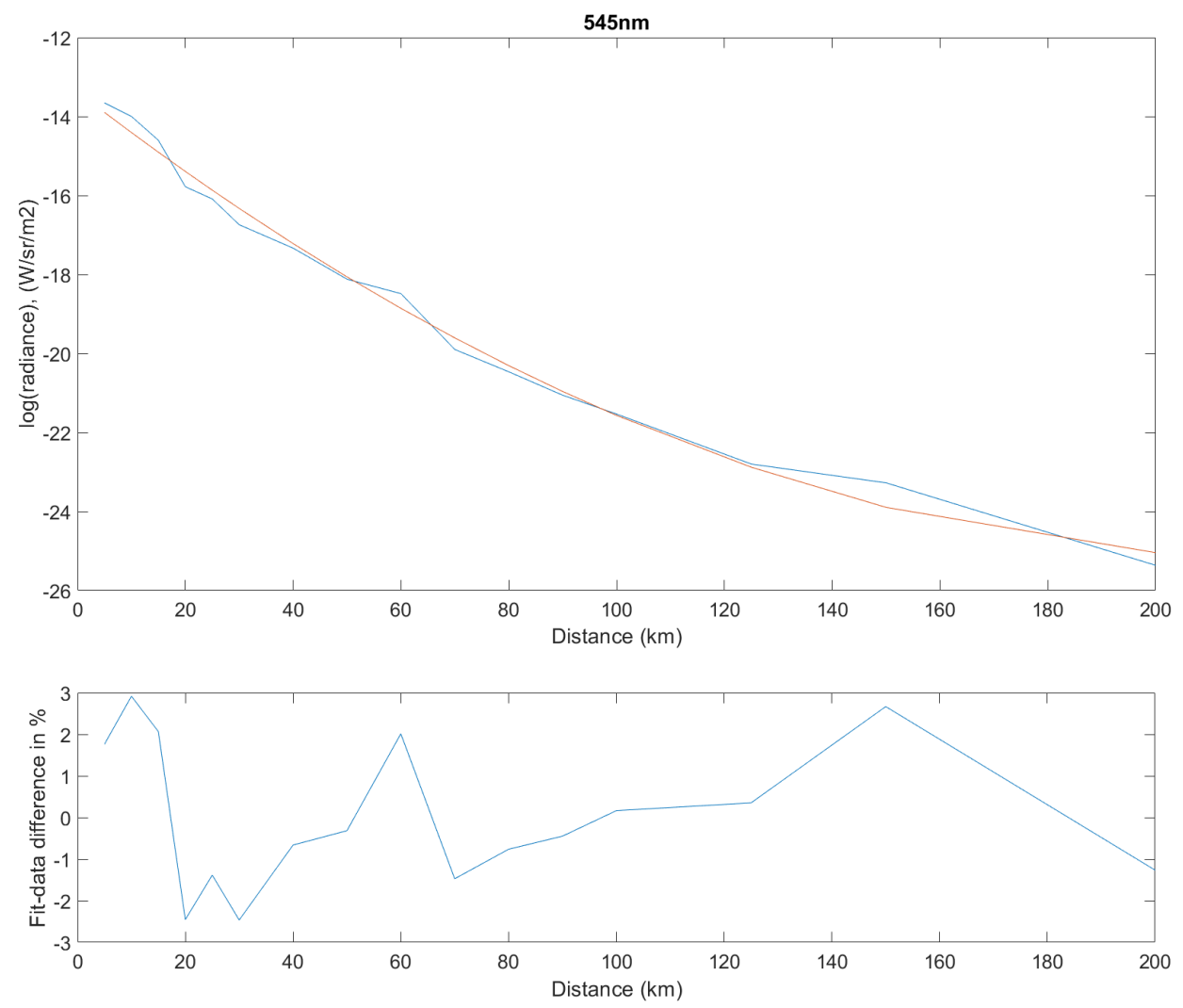

Figure 4: Comparison between the data and the fitting function for the wavelength window centered at $545 \mathrm{~nm}$. Top: data in blue and fitting function in red. Bottom: difference between them in $\%$ following the expression $100 *(\log$ (function) $\log ($ data $)) / \log ($ data $)$

In figure 4 is shown the comparison between the data and expression 2 for the window centered in $545 \mathrm{~nm}$. There are minors differences that confirm the good fit between them. Comparisons in the rest of the spectral windows have a similar behaviour.

\subsection{Adding the light source inventory}

Once obtained the 16 curves and as a last step for obtaining the PSF, the inventory of lights has to be added as a scale factor for each window. This procedure allows to deal with modifications in the inventory of lights by adjusting the scale factors. Typical inventory of the towns around Montsec have been used. Mostly high sodium pressure lamps with a fraction of light emitting diodes of $4000 \mathrm{~K}$ of 


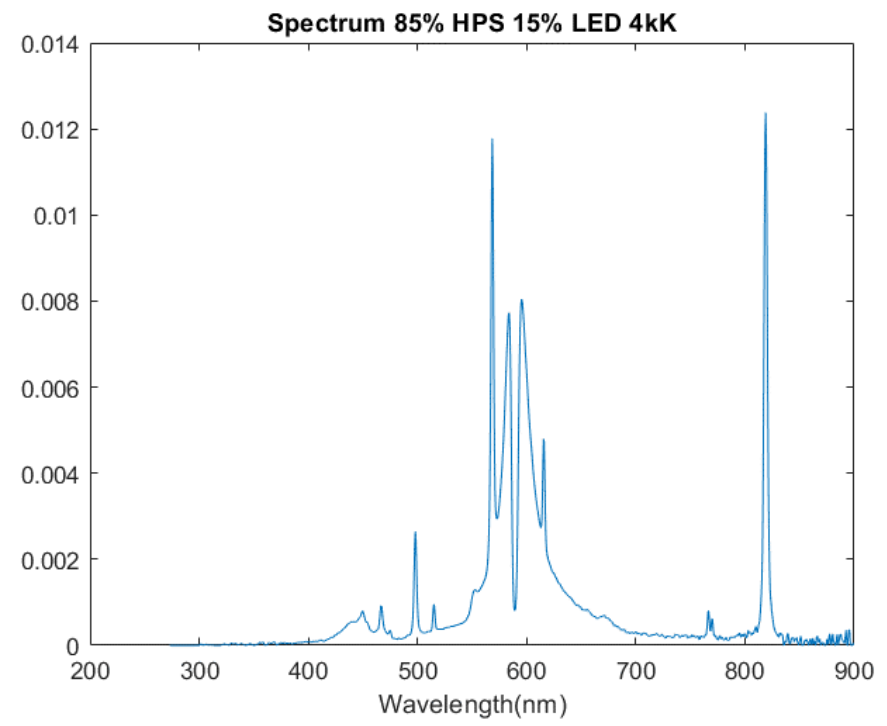

Figure 5: Spectrum of the combination of high pressure sodium lamps and light emitting diode in a relation of luminous flux of $85 \%$ to $15 \%$

color-temperature. Figure 5 shows the shape of the spectrum used.

It is important to remind that the main goal is to find a quick way to determine the major contributors to LP in a specified place. That's the reason why a homogeneous inventory is being used.

\section{Contribution maps by municipality}

In order to decide which sources should be accounted for we divided the territory by municipalities. Normally one municipality include a city or a town, and each one of them is responsible of the lighting within its borders. They are a good reference to sort contributors except for large and divided metropolitan areas. Their division in many municipalities could lead to an underestimation of its contribution, individually they may appear not to be significant but have a noticeable impact when taken as whole. For that reason the metropolitan areas of Barcelona and Tarragona are considered as a unique zone.

The maps show the percentage of the artificial sky brightness received in the PAM (black triangle in the maps) that comes from each one of the municipalities. They are made adding up the contribution of the light sources within each municipality. For further information on how theses maps are created see Bará and Lima (2018). 


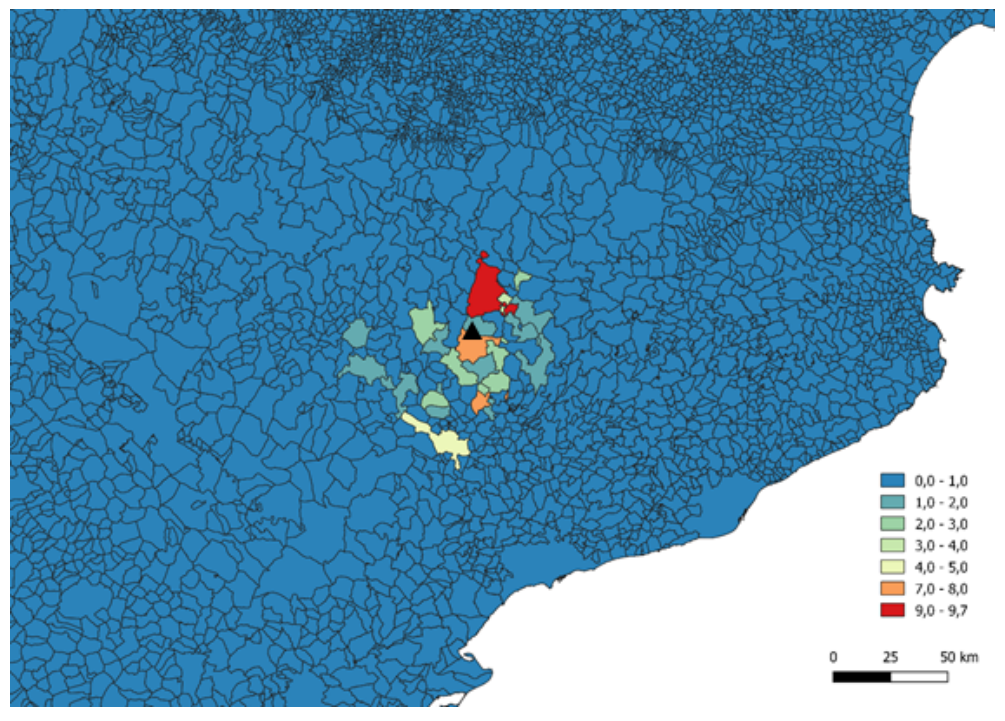

Figure 6: Percentage of artificial light emission by municipality received in the observation point. Filter B

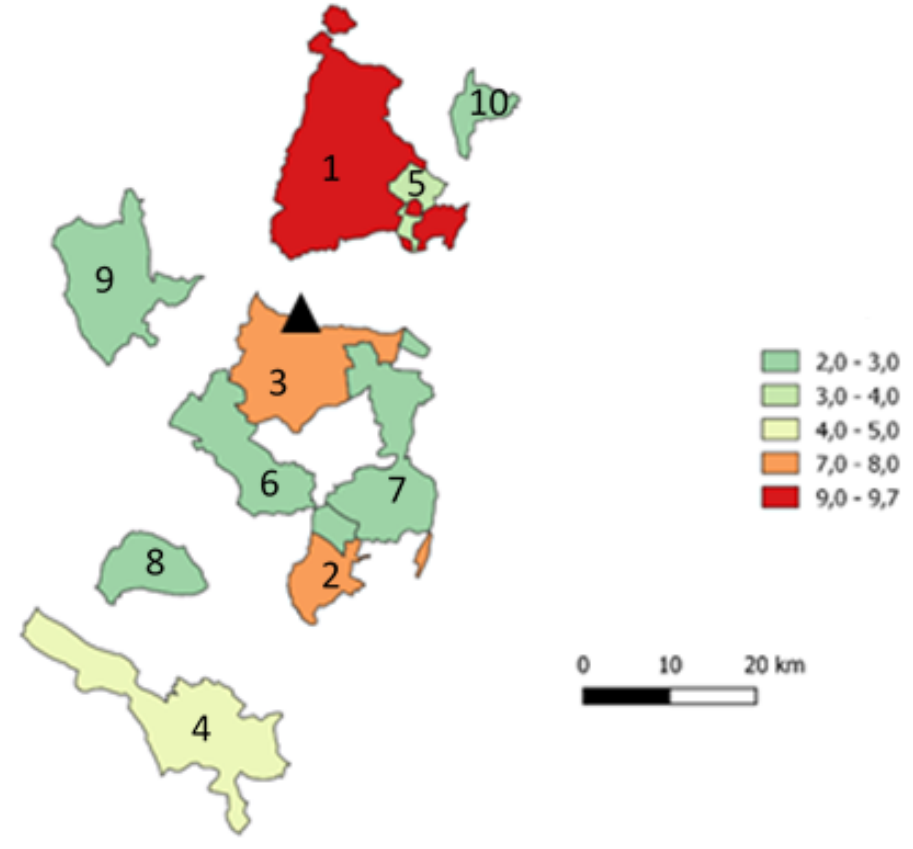

Figure 7: Percentage of artificial light emission by municipality received in the observation point. Filter B over $2 \%$ 


\begin{tabular}{|c|c|c|}
\hline Ranking & Municipality & Contribution to LP (\%) ) \\
\hline 1 & Tremp & 9.66 \\
2 & Balaguer & 7.93 \\
3 & Àger & 7.86 \\
4 & Lleida & 4.73 \\
5 & Talarn & 3.69 \\
6 & Os de Balaguer & 2.45 \\
7 & Camarasa & 2.30 \\
8 & Almenar & 2.29 \\
9 & Benabarre & 2.24 \\
10 & La Pobla de Segur & 2.09 \\
\hline
\end{tabular}

Table 2: Municipalities that contribute more than $2 \%$ to LP in filter B in the sky full dome

\begin{tabular}{|c|c|c|}
\hline Ranking & Municipality & Contribution to LP (\%) ) \\
\hline 1 & Balaguer & 7.31 \\
2 & Tremp & 6.66 \\
3 & Lleida & 6.44 \\
4 & Àger & 3.88 \\
5 & Monzón & 2.66 \\
6 & Talarn & 2.61 \\
7 & Almenar & 2.18 \\
\hline
\end{tabular}

Table 3: Municipalities that contribute more than $2 \%$ to LP in filter V in the sky full dome

\begin{tabular}{|c|c|c|}
\hline Ranking & Municipality & Contribution to LP (\%) ) \\
\hline 1 & Balaguer & 7.09 \\
2 & Lleida & 6.64 \\
3 & Tremp & 6.44 \\
4 & Àger & 3.42 \\
5 & Monzón & 2.74 \\
6 & Talarn & 2.43 \\
7 & Almenar & 2.12 \\
\hline
\end{tabular}

Table 4: Municipalities that contribute more than $2 \%$ to LP in filter R in the sky full dome 


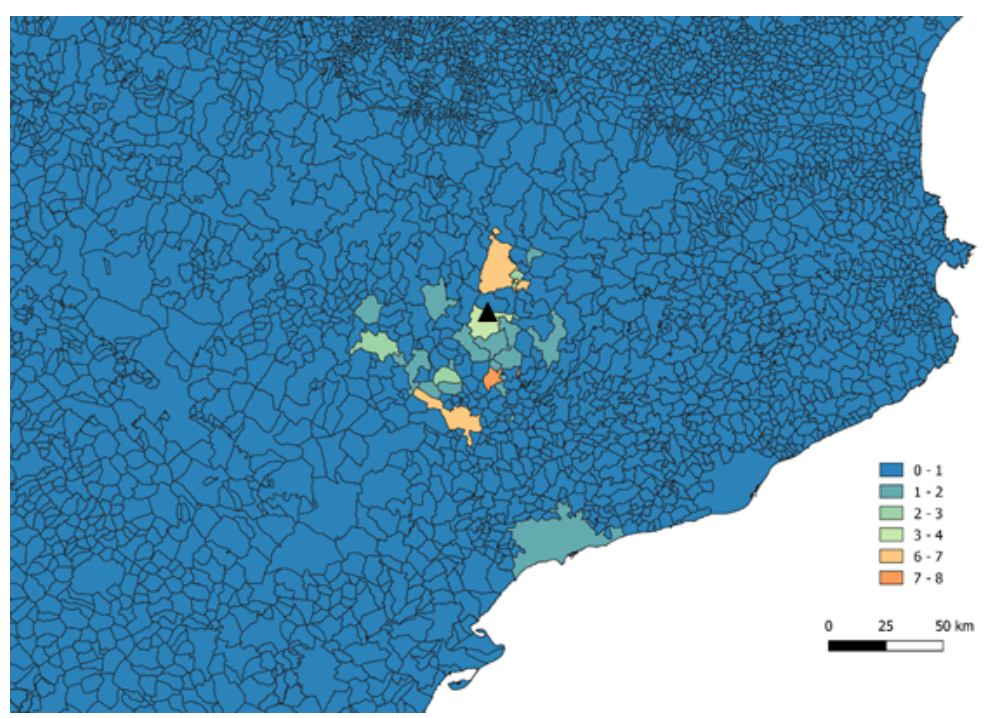

Figure 8: Percentage of artificial light emission by municipality received in the observation point. Filter V

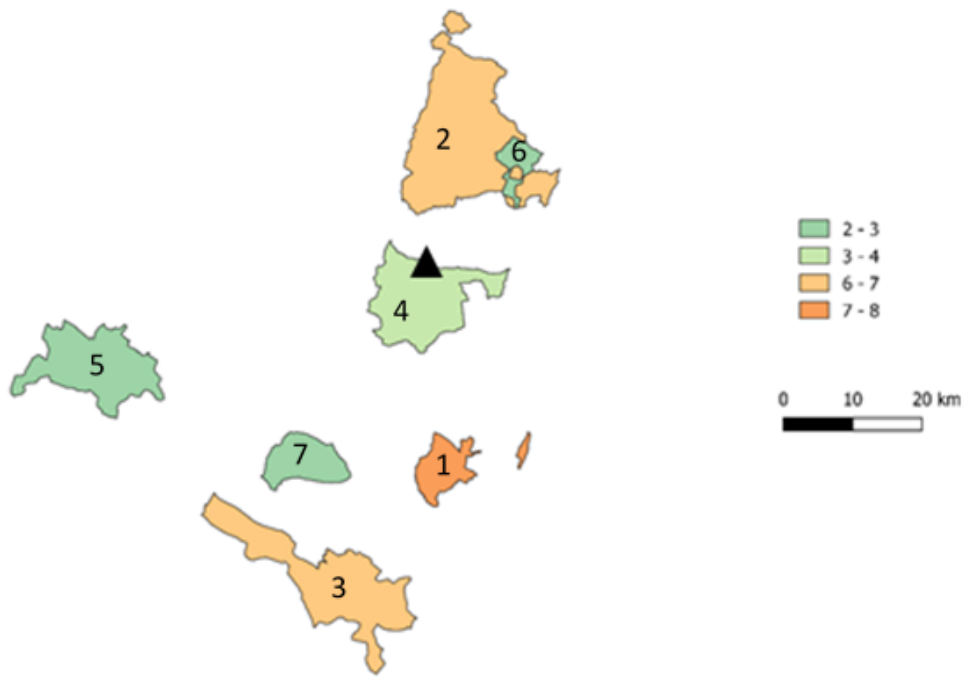

Figure 9: Percentage of artificial light emission by municipality received in the observation point. Filter V over $2 \%$ 


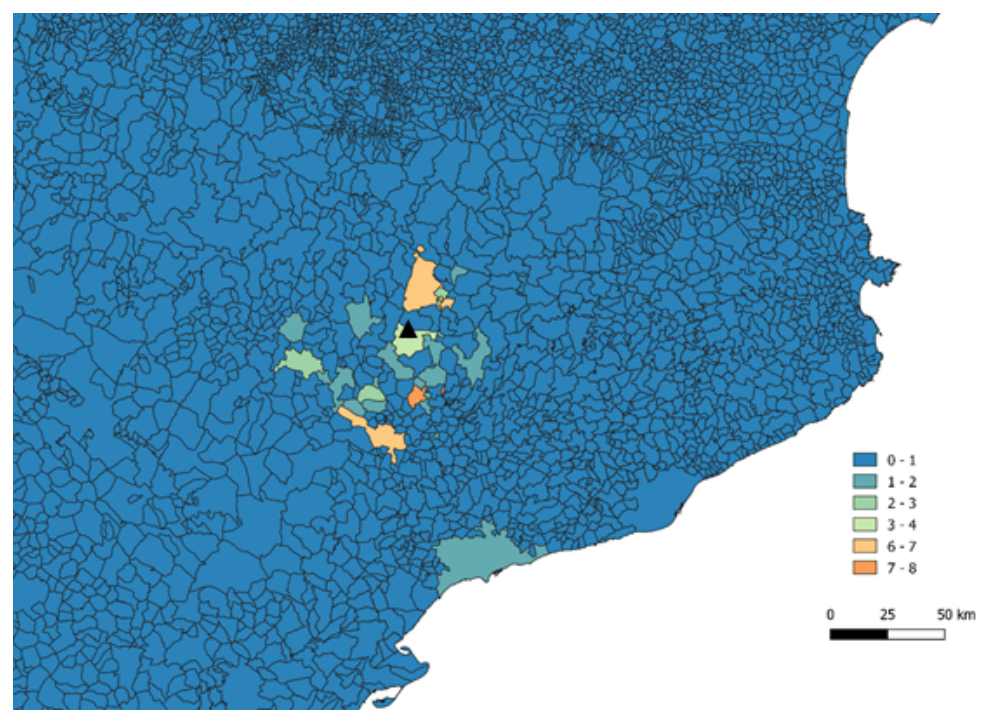

Figure 10: Percentage of artificial light emission by municipality received in the observation point. Filter R

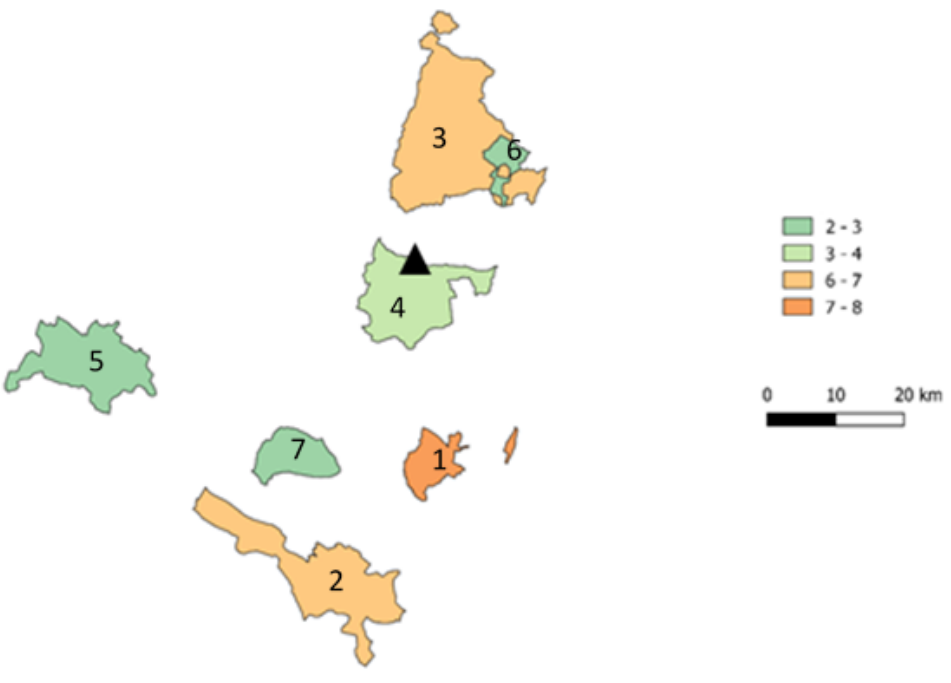

Figure 11: Percentage of artificial light emission by municipality received in the observation point. Filter R over $2 \%$ 


\begin{tabular}{|c|c|c|}
\hline Johnson Filter & Vega (mag) & Natural Sky $\left(\mathrm{mag} / \operatorname{arcsec}^{2}\right)$ \\
\hline B & -0.023 & 22.7 \\
V & -0.023 & 21.8 \\
R & -0.023 & 20.9 \\
\hline
\end{tabular}

Table 5: Apparent magnitudes of Vega and natural sky brightness at zenith.

There are two maps for each filter: on top one with all the municipalities and below other only showing those that contribute more than $2 \%$.

The pattern implicit in the maps correlates with the figure 3, in the sense that in filter B closer light sources are more polluting, even small towns are noticeable. In $\mathrm{R}$, on the other hand, big cities that are placed away from the observer have larger impact than nearby towns. $\mathrm{V}$ is in between these two scenarios.

As stated before, the decision to include more sources contributes to the completeness of the simulations but most of the sources have a negligible contribution that will only lead to wasted CPU time. In our case close to $90 \%$ of the LP comes from light sources within $50 \mathrm{~km}$ from the PAM. Farther sources which contribute more than $2 \%$ has been included as well, specifically the city of Lleida.

\section{All sky brightness maps}

In the previous section we used Illumina v1 to derive a set of PSFs to help us sort which light sources should be included in the simulations of the artificial sky brightness received in the PAM. Now the model is used to compute the total radiance received in the observation point. With the results we can create all sky brightness maps in each filter. These maps show the total radiance received by an observer for any line of sight in the sky dome. They cover from 0 to 90 degrees in elevation and 0 to 360 in azimuth. The simulations can be easily compared with ASTMON and Sky Quality Camera (SQC) instrumentation images and qualitatively with Sky Quality Meter values in order to check its accuracy.

The units used are astronomic magnitudes, that are a dimensionless logarithmic scale. They are described by:

$$
m=m_{\text {ref }}-2.5 \log _{10}\left(\frac{F}{F_{r e f}}\right)
$$

$m_{\text {ref }}$ is the magnitude of a reference source with an irradiance $F_{r e f}$. Vega star has been used as a reference source. 
Total radiance includes artificial and natural sources, as Illumina provides artificial radiance the natural radiance is added using a reference value (Walker, 1987) of $22.7 \mathrm{mag} / \mathrm{arcsec}^{2}$ in B, $21.8 \mathrm{mag} / \operatorname{arcsec}^{2}$ in $\mathrm{V}$ and $20.9 \mathrm{mag} / \mathrm{arcsec}^{2}$ in R. Bear in mind that in the absence of artificial radiance those would be the values present in the map.

\subsection{Model parameters}

Atmospheric conditions are the same than the ones used for deriving the PSF, see section3.

From the information revealed by the previous section, 4 different light zones will be included in the calculus, as shown in figure 12 .

- Circular zone centered in the PAM with $50 \mathrm{~km}$ of radius, that includes almost all the lights that are significantly contributing to the LP received in every filter. The inventory of lights of this zone is the most common among the towns within, that coincides with the one used for determining the PSF: $85 \%$ HPS with ULOR between 5 and 10\%; and 15\% LED $4000 \mathrm{~K}$ with ULOR of $1 \%$

- The city of Lleida that is partially outside the big circular zone and have a different light sources inventory: 74\% HPS with ULOR 0\% and 10\%; 14\% LED4000k with ULOR $0 \%$; and $12 \%$ metal hallide $(\mathrm{MH})$ with ULOR $0 \%$.

- The city of Balaguer that is within the big zone but due to its big contribution and its special inventory of lights is defined separately: $86 \%$ HPS with ULOR $0,5,15$ and $20 \% ; 11 \% \mathrm{MH}$ with ULOR $0 \%$; and $2 \%$ LED4000K with ULOR $0 \%$.

- The city of Tremp because of its contribution and the lack of LED in its inventory: $100 \%$ HPS with different ULOR, from $0 \%$ to $20 \%$.

There are other sources, as Àger, placed inside the first zone that are main contributors. Their inventories are very similar to the one defined by the big zone and simulations show no difference if they are defined separately or within the big zone. For the sake of simplification it has been decided to include them in the big zone.

Each zone overwrite the previous one, in the sense that they are not doubled or their inventories mixed. 


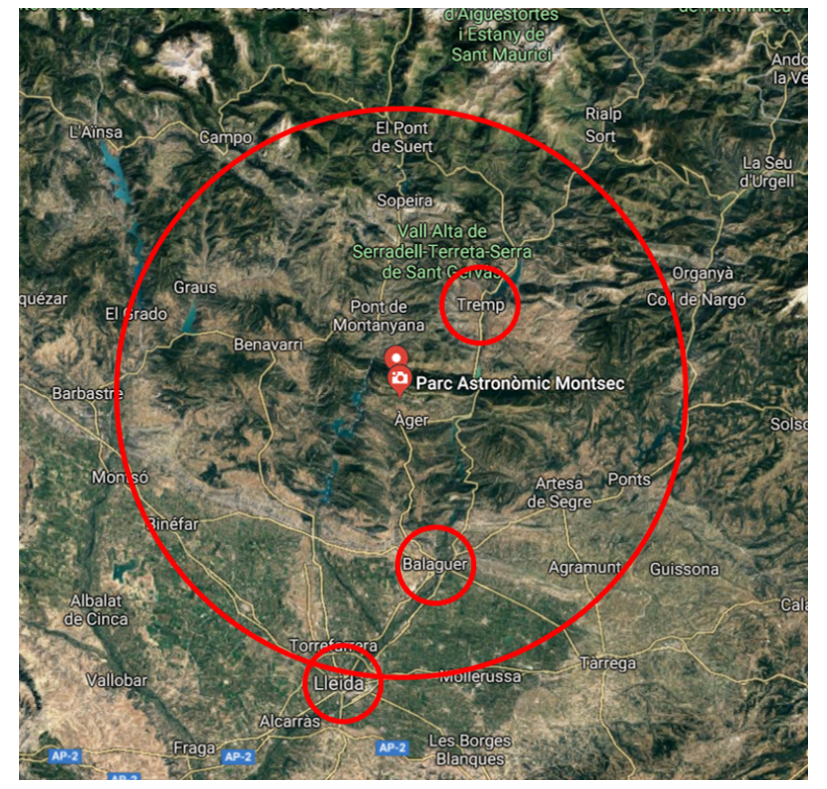

Figure 12: Scheme of the area included in the simulation with its different zones.

\subsection{Results}

The validation process of the results is based in the comparison with the data obtained from measurements obtained with ASTMON, SQM and SQC.

The All-Sky Transmission Monitor (ASTMON) is based on a $\mathrm{f}=4.5 \mathrm{~mm}$ fisheye lens and an integrated astronomical CCD camera. It measures the luminance of the all night sky in several wavelength bands. The system is designed to perform a continuous monitoring of the surface brightness of the night sky background in a fully robotic mode. In addition to the sky background brightness, ASTMON can provide atmospheric extinction and cloud coverage estimates for the entire sky surface at the same time (see Aceituno et al. (2011) and Hänel et al. (2017)). This device allowed to validate results in the three filters studied, $\mathrm{B}, \mathrm{V}$ and $\mathrm{R}$.

Sky Quality Meter (SQM) is the most spread photometer in the light pollution community, mainly because of its low cost. It is produced by Unihedron company (Grimsby, ON, Canada). It is equipped with a solid state detector (RAOS TSL237s) combined with a light-frequency converter and a filter(HOYA CM-500) that makes it sensible to the range between 320 and 720nm (see figure 13). Althogh not the same it is similar to $\mathrm{V}$ filter. Its field of view is limited to a portion of the sky, it is used normally pointing to the zenith, see figure 14 .

Sky Quality Camera is a commercial image processing software (Euromix, Slove- 


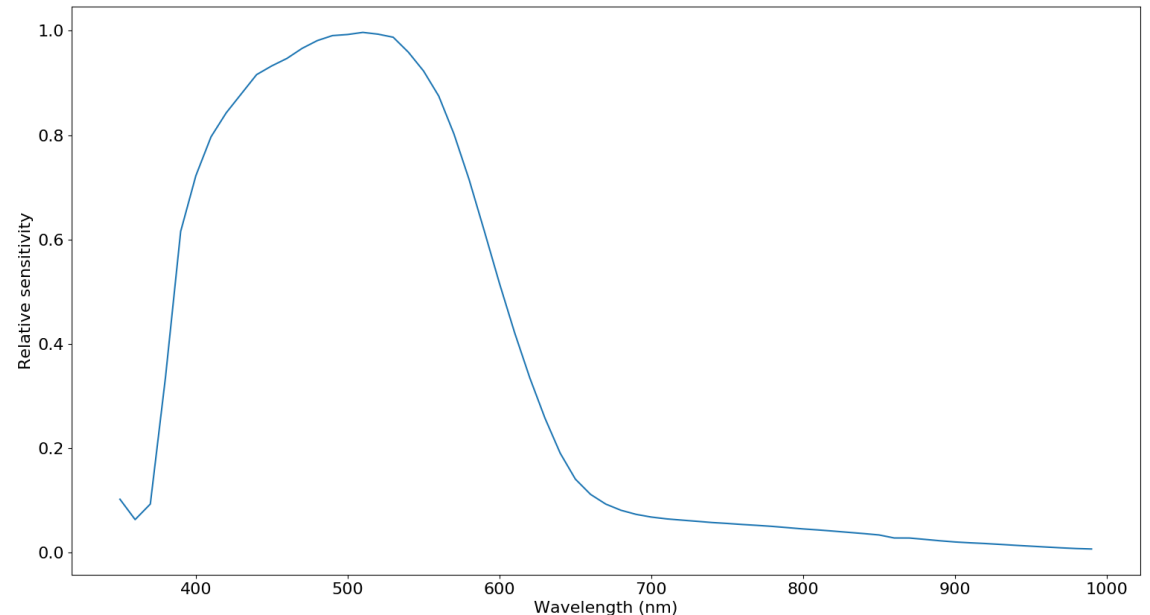

Figure 13: SQM's sensitivity with respect to the wavelength (Bará et al., 2019)

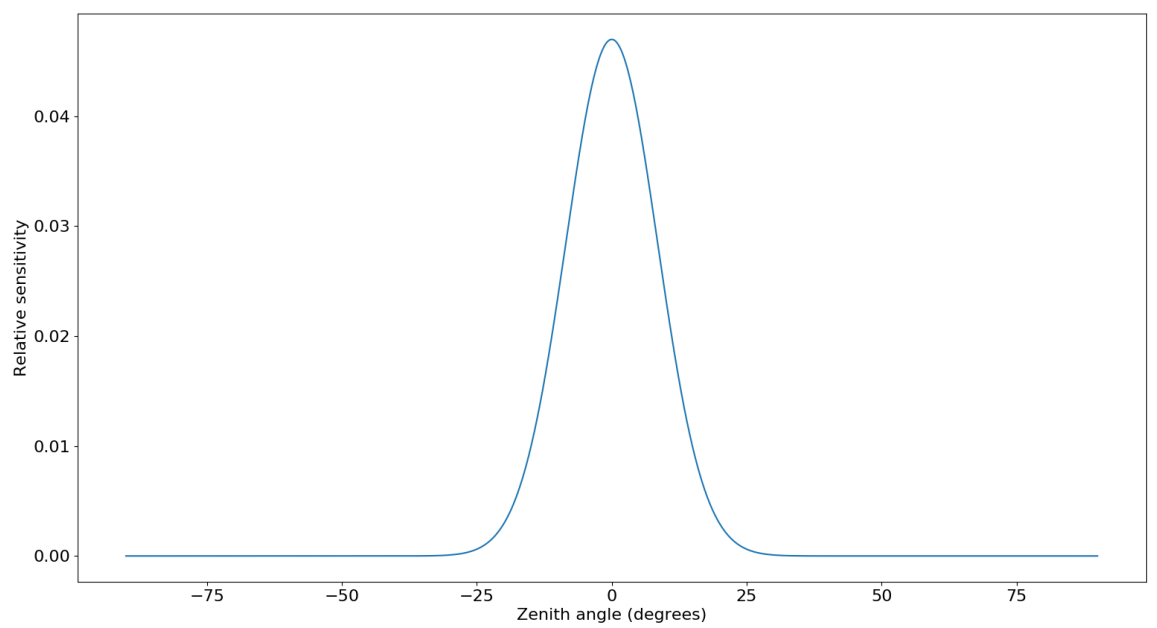

Figure 14: SQM's sensitivity as function of the angle from the center of the field of view. It has a lens which establishes its 20 full width at half maximum (FWHM) field of view (SQM operator's manual, Unihedron) 
nia). The camera has to be calibrated by the manufacturer. The calibration only covers the green channel of the camera so it is only possible to see data in the $\mathrm{V}$ filter. The camera used in this case is a commercial DSLR camera, a Canon EOS 6D, which has a full-frame CMOS sensor with 20.2 Megapixel (5496 x 3670 pixels). The camera has a built-in GPS sensor, and allows ISO settings from 100 to 25,600 and shutter speed ranges down to $30 \mathrm{~s}$ in automatic mode. We operated the camera with a circular fisheye lens (Sigma EX DG with $8 \mathrm{~mm}$ focal length) always at full aperture of 3.5 .

Simulations are in great agreement with ASTMON and SQC measurements (figures $15,16,17,19)$. When comparing the $\mathrm{V}$ filter results with SQC data bear in mind that the E-W axis is switched. The Milky Way is clearly visible in measurements but it is not included in the simulations, it is responsible for the slightly non-symmetrical pattern around the zenith that is present in observations.

The geographical pattern of brightness in each filter is almost identical, light pollution came from the same directions and affects very similar ranges of elevation and azimuth. The values in all the dome are very similar for each filter with differences typically below $0.2 \mathrm{mag} / \operatorname{arcsec}^{2}$. Simulations and measurements agree on showing a extraordinary dark sky. Above 30-35 degrees values are less than $0.3 \mathrm{mag} / \mathrm{arcsec}^{2}$ brighter than the reference used as maximum for a natural sky $\left(22.7 \mathrm{mag} / \mathrm{arcsec}^{2}\right.$ in $\mathrm{B}, 21.8 \mathrm{mag} / \operatorname{arcsec}^{2}$ in $\mathrm{V}$ and $20.9 \mathrm{mag} / \operatorname{arcsec}^{2}$ in R). Notice that in R filter ASTMON data close to the zenith goes beyond the reference maximum that we imposed for simulations $\left(>21.0 \mathrm{mag} / \operatorname{arcsec}^{2}\right)$. In a similar way the darkest value in SQC data is $>21.9 \mathrm{mag} / \operatorname{arcsec}^{2}$ and in the simulations $21.6 \mathrm{mag} / \mathrm{arcsec}^{2}$, the difference is quite noticeable but as stated the reference darkest sky is $21.8 \mathrm{mag} / \operatorname{arcsec}^{2}$, so either the reference has to be reconsidered or if confirmed as valid the SQC data has to be corrected.

ASTMON, SQC and simulations agree in the most polluting directions, south and south-east that matches with the directions to Lleida, Balaguer and Àger. Tremp is also noticeable in the north-east direction although less in B, its inventory is made only by HPS that has almost no blue light. There is a dimmer light pollution source that can be noticed (mostly in $\mathrm{R}$ ) in measurements but not in simulations, east south-east (close to 120 degrees) pointing to Barcelona area. On that night there where high clouds over Barcelona that increased the spread of its light pollution, in the map of municipalities (section 4) clear sky was considered and Barcelona showed negligible contribution and has not been included as a light source. Another mismatch is the brightness coming from west south-west that points to the city of Monzon and Zaragoza further away, in the simulations only Monzon is studied, that's probably the reason this little bright dome is more noticeable than in simulations. 

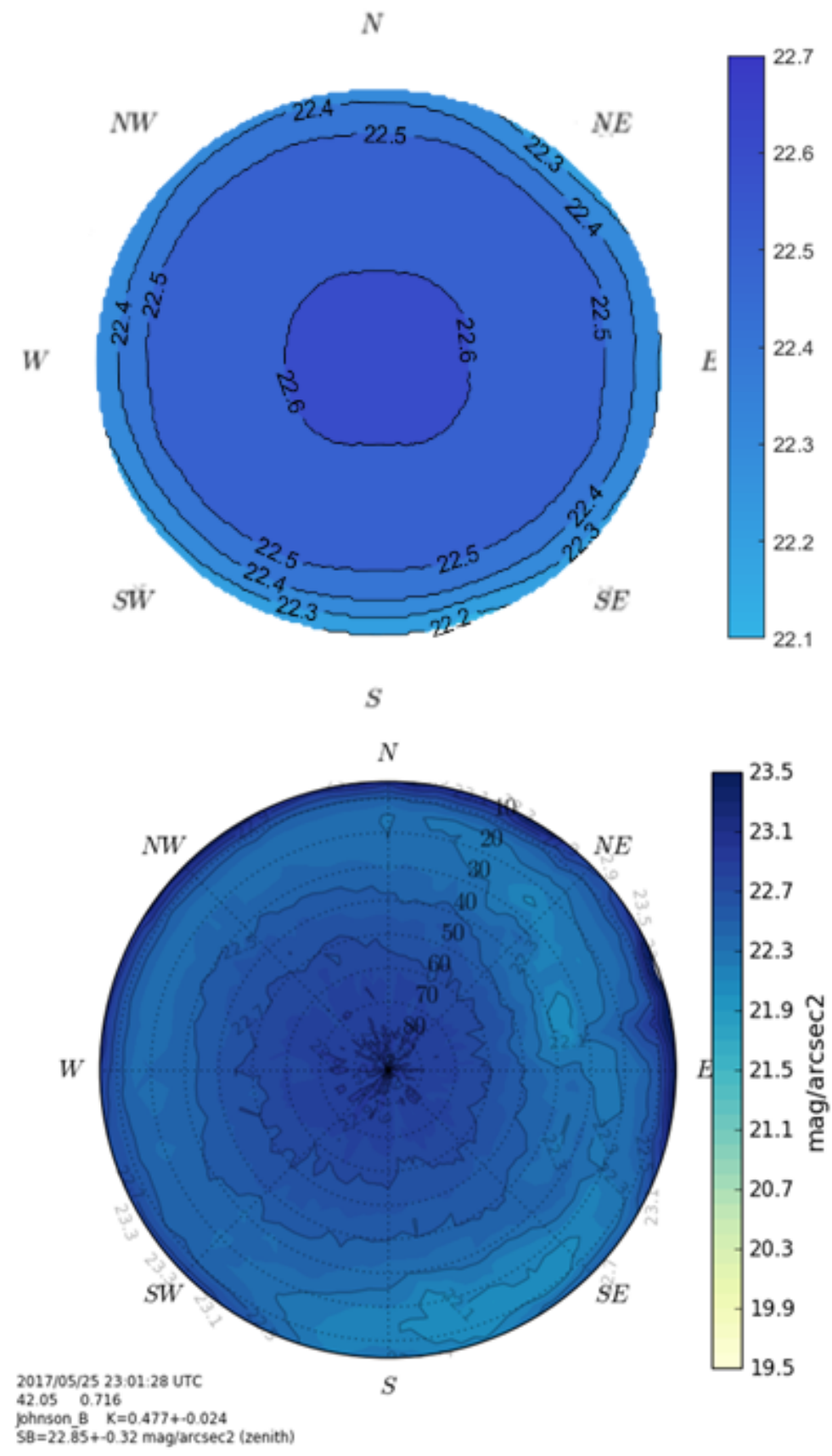

Figure 15: All sky brightness maps in B. Top: simulation, 21.61mag/ $\operatorname{arcsec}^{2}$ at zenith. Bottom: ASTMON data. 

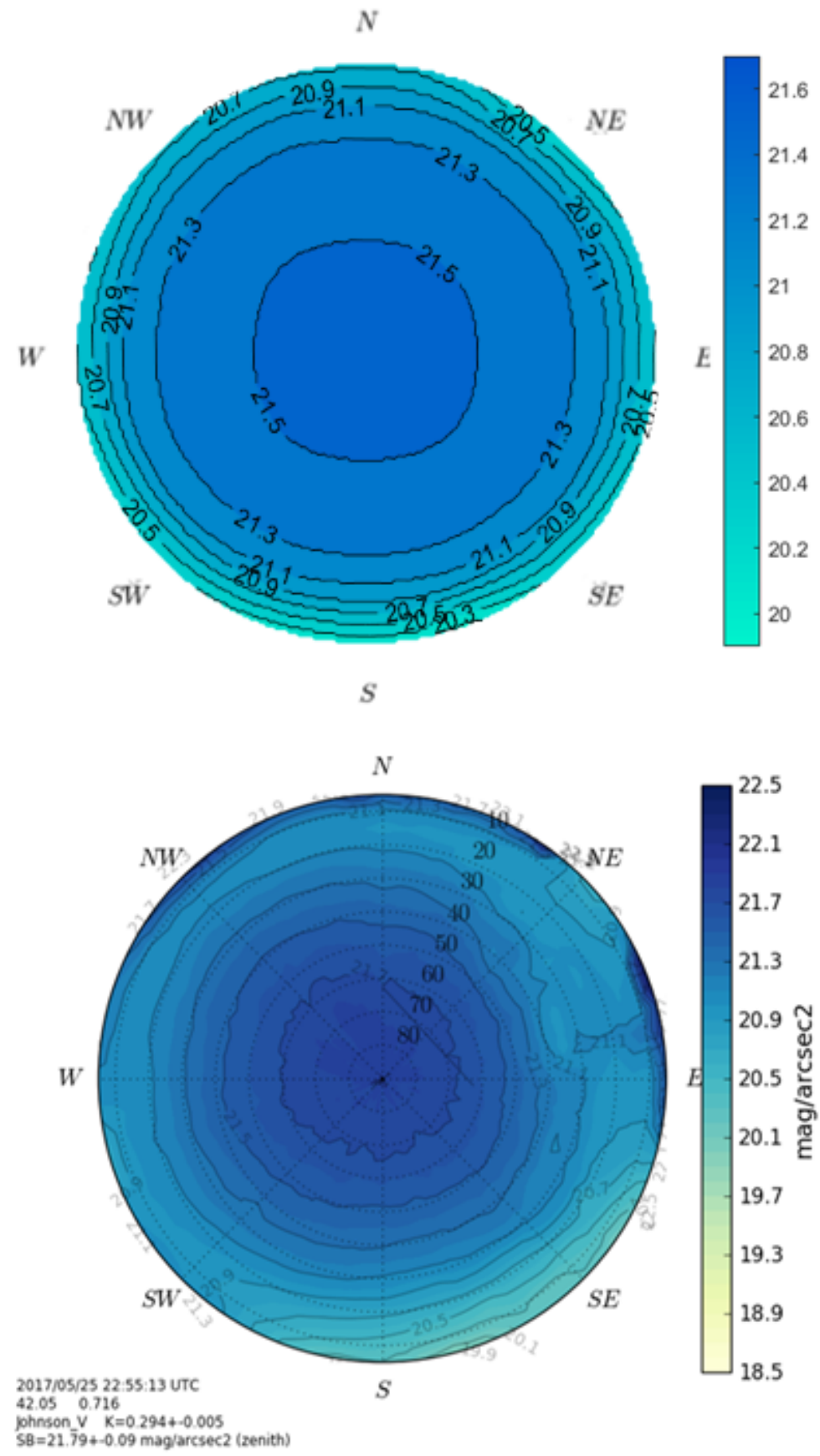

Figure 16: All sky brightness maps in V. Top: simulation, $21.52 \mathrm{mag} / \operatorname{arcsec}^{2}$ at zenith. Bottom: ASTMON data. 


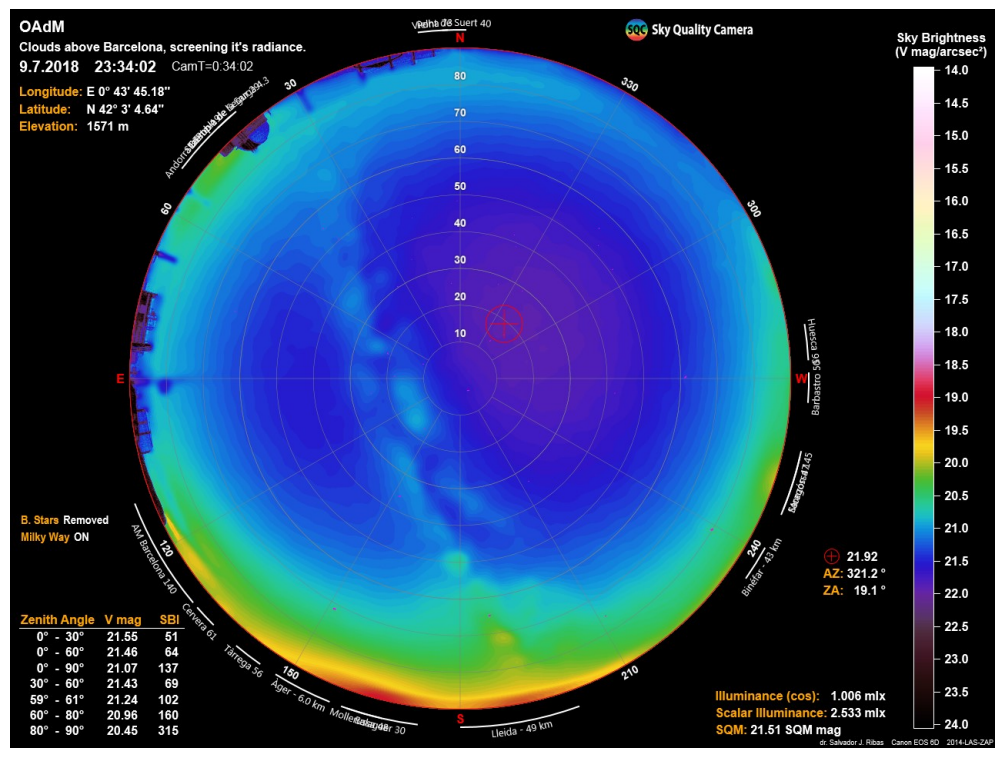

Figure 17: SQC data from PAM. 25th May 2017.

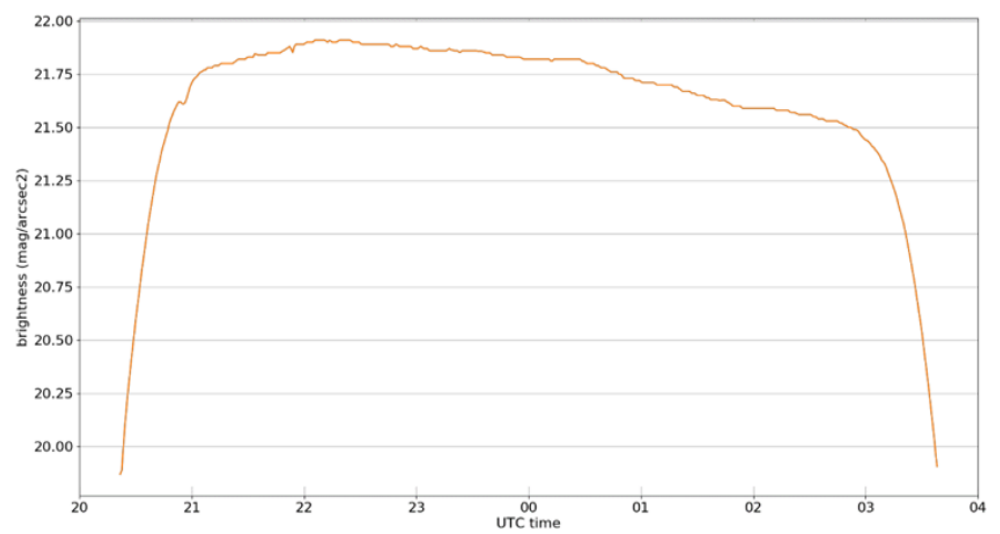

Figure 18: SQM data from PAM. 25th May 2017. 


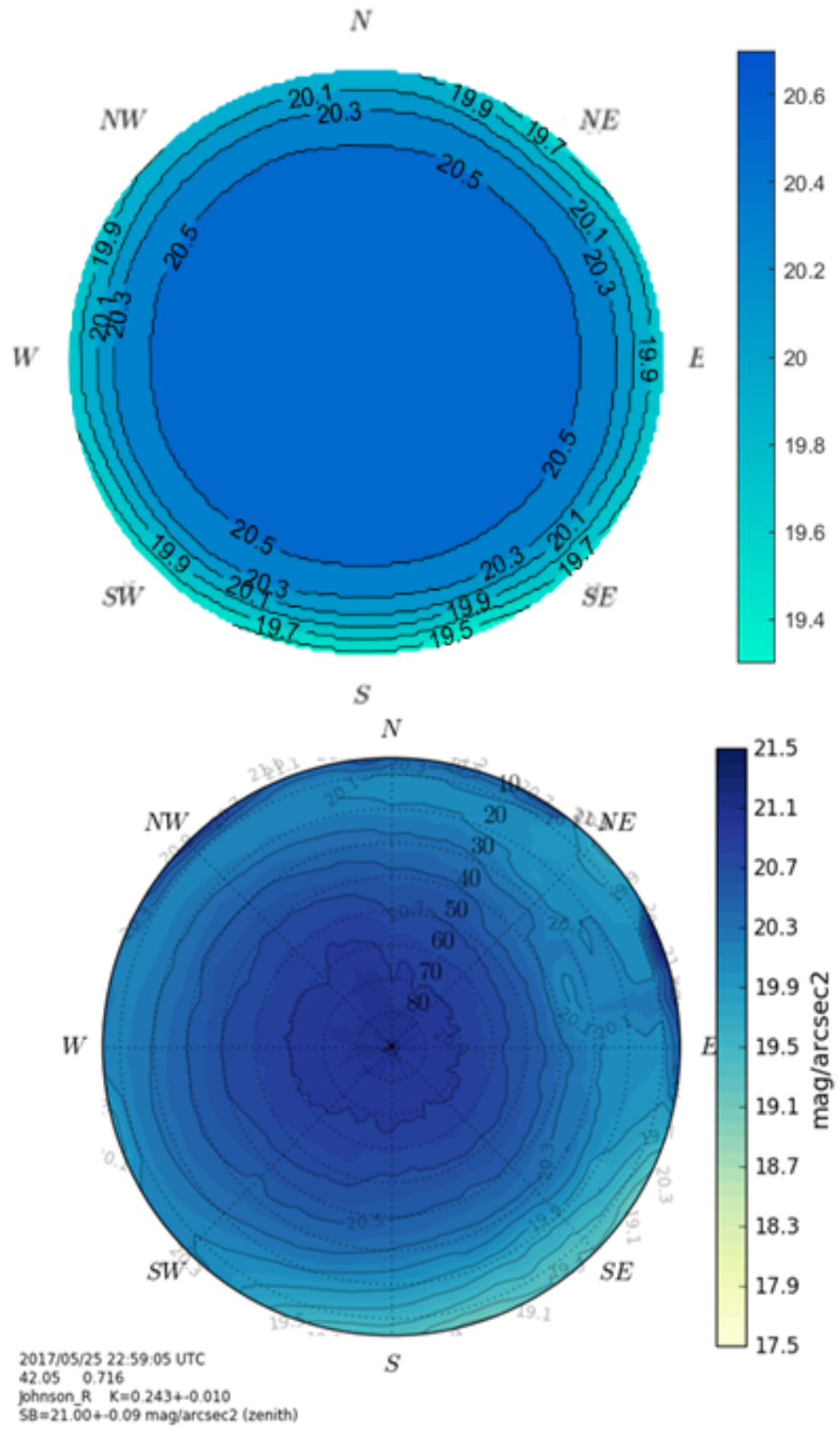

Figure 19: All sky brightness maps in R. Top: simulation, $20.69 \mathrm{mag} / \operatorname{arcsec}^{2}$ at zenith. Bottom: ASTMON data. 
SQM data confirms the quality of the night sky in the observatory. But it is hard to compare it with simulations or even other data. SQC software estimates what should be the reading of a SQM from the picture it is processing and the estimation is $21.51 \mathrm{mag} / \operatorname{arcsec}^{2}$ that is far from the $21.85 \mathrm{mag} / \operatorname{arcsec}^{2}$ that is measured by SQM when the picture was taken.

\section{Conclusions}

In this paper night sky brightness over Montsec Observatory was simulated successfully using Illumina numerical model. This study focused on reproducing measurements to ensure the validation of the methodology presented. Satisfactory levels of correlation have been achieved between simulations and measurements. However, the presence of clouds is not studied and the methodology is not yet validated beyond clear sky conditions. Considering this it can be used in other locations with no measurements and as a prediction tool for changes in lighting systems.

In the process a new application for Illumina has been proposed: deriving simplified PSFs to estimate which areas (municipalities, regions, counties, etc.) are polluting more the location we want to study. This approach is helpful when deciding which sources should be included in the simulations. The same PSFs can be used in places with similar atmospheric conditions. If the inventory of lights is different from the one proposed in this paper, it is simple to get the correct one by changing the scale factors related with the spectrum. In places with different atmospheric conditions (humidity, pressure, aerosol optical depth, etc.) the whole process should be performed from scratch. Nevertheless, it reduces the time needed to achieve an accurate simulation of the night sky brightness by knowing beforehand that the principal stakeholders are taken into account. Without this process researchers have to rely on intuition, this leads to possible drawbacks: 1)including negligible sources that increase the CPU time, and 2)ignoring important sources. In our case more than $95 \%$ of the estimated artificial radiance comes from nearby towns within $50 \mathrm{~km}$ from the observatory alongside with the city of Lleida further away.

With this information we computed the artificial radiance received in the observatory using Illumina v1. This information allowed us to create virtual all-sky brightness maps over the observatory and compare them to measurements taken with ASTMON, SQC and SQM instumentation. The typical deviation is below 0.2 $\mathrm{mag} / \operatorname{arcsec}^{2}$ that is of the order of magnitude of the variations observed due to atmospheric condition, Milky Way presence and other phenomena. Contribution maps by municipalities, simulations and measurements reveal that there are 4 main light pollution sources that affect the sky over the PAM: the city of Lleida of 140k inhabitants 
located at $50 \mathrm{~km}$ to the south (180-190), the city of Balaguer of 20k inhabitants located at $30 \mathrm{~km}$ to the south-eat (150-160), the city of Tremp of $6 \mathrm{k}$ inhabitants located at $20 \mathrm{~km}$ to the north-east (40-50) and the nearby town of Àger of 700 inhabitants located at less than $5 \mathrm{~km}$ to the south (170).

\section{Bibliography}

Aceituno, J., Sánchez, S., Aceituno, F., Galadí-Enríquez, D., Negro, J., Soriguer, R., Gomez, G. S., 2011. An all-sky transmission monitor: Astmon. Publications of the Astronomical Society of the Pacific 123 (907), 1076.

Aubé, M., 2007. Light pollution modeling and detection in a heterogeneous environment. In: Proceedings of Starlight 2007 conference, La Palma, Spain.

Aubé, M., Franchomme-Fossé, L., Robert-Staehler, P., Houle, V., 2005. Light pollution modelling and detection in a heterogeneous environment: toward a night-time aerosol optical depth retreival method. In: Atmospheric and Environmental Remote Sensing Data Processing and Utilization: Numerical Atmospheric Prediction and Environmental Monitoring. Vol. 5890. International Society for Optics and Photonics, p. 589012.

Aubé, M., Kocifaj, M., 2012. Using two light-pollution models to investigate artificial sky radiances at canary islands observatories. Monthly Notices of the Royal Astronomy Society 422(1), 819-830.

Aubé, M., Simoneau, A., Wainscoat, R., Nelson, L., 2018. Modelling the effects of phosphor converted led lighting to the night sky of the haleakala observatory, hawaii. Monthly Notices of the Royal Astronomical Society 478, 1776-1783.

Bará, S., Lima, R., 2018. Photons without borders: quantifying light pollution transfer between territories. International Journal of Sustainable Lighting 20(2), 51-61.

Bará, S., Tapia, C., Zamorano, J., 2019. Absolute radiometric calibration of tess-w and sqm night sky brightness sensors. Sensors 19(6), 1336.

Cousins, A., 1976. Standard stars for vri photometry with s25 response photocathodes. Monthly Notes of the Astronomical Society of South Africa 35, 70.

Duriscoe, D. M., 2016. Photometric indicators of visual night sky quality derived from all-sky brightness maps. Journal of Quantitative Spectroscopy and Radiative Transfer 181, 33-45. 
Duriscoe, D. M., Anderson, S. J., Luginbuhl, C. B., Baugh, K. E., 2018. A simplified model of all-sky artificial sky glow derived from viirs day/night band data. Journal of Quantitative Spectroscopy and Radiative Transfer 214, 133-145.

Garcia-Saenz, A., Sánchez de Miguel, A., Espinosa, A., Valentin, A., Aragonés, N., Llorca, J., et al., 2018. Evaluating the association between artificial light-at-night exposure and breast and prostate cancer risk in spain (mcc-spain study). Environ Health Perspect. 126(4).

Gueymard, C., 2001. Parameterized transmittance model for direct beam and circumsolar spectra irradiance. Solar Energy 71(5), 325-346.

Hänel, A., Posch, T., Ribas, S. J., Aubé, M., Duriscoe, D., Jechow, A., Kollath, Z., Lolkema, D. E., Moore, C., Schmidt, N., et al., 2017. Measuring night sky brightness: methods and challenges. Journal of Quantitative Spectroscopy and Radiative Transfer.

Holben, B. N., Eck, T. F., Slutsker, I., Tanre, D., Buis, J., Setzer, A., Vermote, E., Reagan, J., Kaufman, Y., Nakajima, T., et al., 1998. Aeroneta federated instrument network and data archive for aerosol characterization. Remote sensing of environment 66 (1), 1-16.

Jechow, A., Kollath, Z., Ribas, S. J., Spoelstra, H., Holker, F., C, . K. C., 2017. Imaging and mapping the impact of clouds on skyglow with all-sky photometry. Scientific reports 7(1).

Jechow, A., Ribas, S. J., Domingo, R. C., Holker, F., Kollath, Z., Kyba, C. C., 2018. Tracking the dynamics of skyglow with differential photometry using a digital camera with fisheye lens. Journal of Quantitative Spectroscopy and Radiative Transfer 229, 212-223.

Johnson, H., Morgan, W., 1953. Fundamental stellar photometry for standards of spectral type on the revised system of the yerkes spectral atlas. The Astrophysical Journal 117, 313.

Johnson, H., Morgan, W., 1955. The ubv photometric system. Ann. Astrophys 18, 292-296.

Kocifaj, M., 2007. Light-pollution model for cloudy and cloudless night skies with ground-based light sources. Applied optics 46 (15), 3013-3022. 
Linares, H., Masana, E., Ribas, S., Garcia-Gil, M., Aubé, M., Figueras, F., 2018. Modelling the night sky brightness and light pollution sources of montsec protected area. Journal of Quantitative Spectroscopy and Radiative Transfer 217, 178-188.

Walker, A., 1987. NOAO newsletter 10, 16. 